\title{
Democratizing Al: Non-expert design of prediction tasks
}

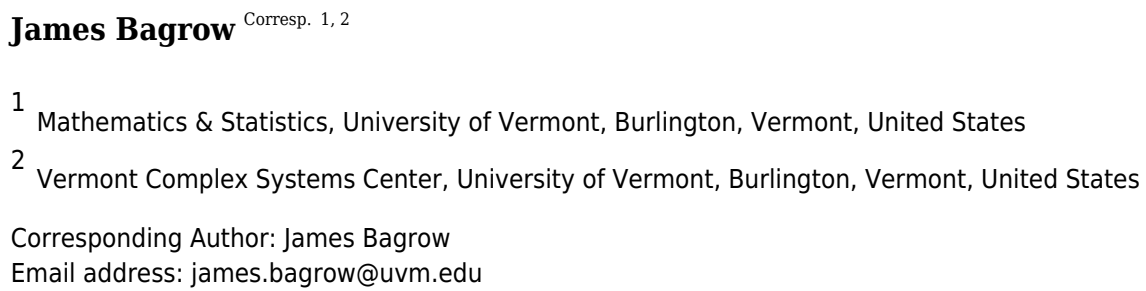

Non-experts have long made important contributions to machine learning (ML) by contributing training data, and recent work has shown that non-experts can also help with feature engineering by suggesting novel predictive features. However, non-experts have only contributed features to prediction tasks already posed by experienced ML practitioners. Here we study how non-experts can design prediction tasks themselves, what types of tasks non-experts will design, and whether predictive models can be automatically trained on data sourced for their tasks. We use a crowdsourcing platform where non-experts design predictive tasks that are then categorized and ranked by the crowd. Crowdsourced data are collected for top-ranked tasks and predictive models are then trained and evaluated automatically using those data. We show that individuals without ML experience can collectively construct useful datasets and that predictive models can be learned on these datasets, but challenges remain. The prediction tasks designed by non-experts covered a broad range of domains, from politics and current events to health behavior, demographics, and more. Proper instructions are crucial for non-experts, so we also conducted a randomized trial to understand how different instructions may influence the types of prediction tasks being proposed. In general, understanding better how non-experts can contribute to ML can further leverage advances in Automatic ML and has important implications as ML continues to drive workplace automation. 


\title{
Democratizing Al: Non-expert design of prediction tasks
}

\author{
James P. Bagrow ${ }^{1,2}$ \\ ${ }^{1}$ Mathematics \& Statistics, University of Vermont, Burlington, Vermont, United States \\ ${ }^{2}$ Vermont Complex Systems Center, University of Vermont, Burlington, Vermont, United \\ States \\ Corresponding author: \\ James P. Bagrow \\ Email address: james.bagrow@uvm.edu
}

10 ABSTRACT

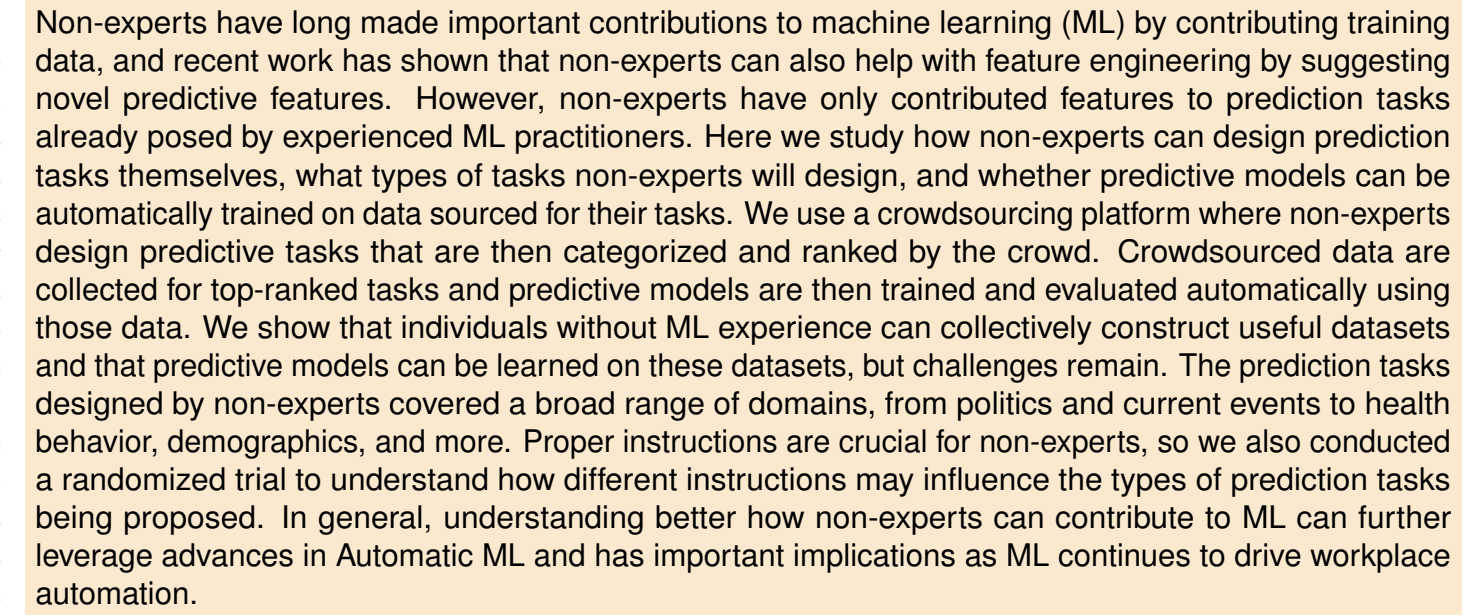

\section{INTRODUCTION}

Recent years have seen improved technologies geared towards workplace automation and there is both promise and peril in how AI, robotics, and other technologies can alter the job security and prospects of the future workforce (David, 2015; Frank et al., 2019). While automation has already upended factory work and other traditionally blue collar jobs, machine learning (ML) can have similar effects on offices and knowledge work. ML can enable firms to better understand and act on their data more quickly and perhaps with fewer employees. But how well can or should employees understand the process and scope of ML? Perhaps most importantly, on their own, can individuals apply ML in new problem areas, informed by their own domain knowledge, or is such "editorial" control of ML limited to experts with significant training and experience in ML and related areas?

At the same time, machine learning itself is being automated (Feurer et al., 2015), and the emerging field of Automatic Machine Learning promises to lower the barrier of entry at least to some extent, and in time the role of ML expertise may be supplanted by sufficiently advanced "AutoML" methods. Yet non-experts (meaning those with little prior experience in ML) will not be able to apply ML to a new area of their own interest if they can only contribute features to pre-existing problems in pre-existing areas. To fully leverage advances in ML, non-experts must be able to define their own tasks in new areas.

In this paper, we investigate the following research questions (RQs):

1. Can individuals who are not experts in the details of statistical or machine learning design meaningful supervised learning prediction tasks? 
2. What are the properties of prediction tasks proposed by non-experts? Do tasks tend to have common properties or focus on particular topics or types of questions?

3. Does providing an example of a prediction task help clarify the design assignment for non-experts or do such examples introduce bias?

4. Are non-experts able to compare and contrast new prediction tasks, to determine which tasks should be deemed important or interesting?

5. Can data collected for proposed tasks be used to build accurate predictive models without intervention from an ML expert?

To study these questions, we employ a crowdsourcing platform where groups of crowd workers ideate supervised learning prediction tasks (RQ1), categorize and efficiently rank those tasks according to criteria of interest (RQ4), and contribute training data for those tasks. We study the topics and properties of prediction tasks (RQ2), show that performant predictive models can be trained on some proposed tasks (RQ5), and explore the design of the problem of proposing prediction tasks using a randomized trial (RQ3). We discuss limitations and benefits when approaching learning from this perspective-how it elevates the importance of specifying prediction task requirements relative to feature engineering and modeling that are traditionally the focus of applied machine learning.

\section{BACKGROUND}

Machine learning (ML) requires experts to understand technical concepts from probability and statistics, linear algebra, and optimization; be able to perform predictive model construction, training, and diagnostics; and even participate in data collection, cleaning, and validation (Domingos, 2012; Alpaydin, 2020). Such a depth of pre-requisite knowledge may limit the roles of non-experts, yet fields such as automatic machine learning (AutoML) (Hutter et al., 2019) have the promise to further enable non-expert participation in ML by removing many of the challenges which non-experts may be unable to address without training or experience (Feurer et al., 2015; Vanschoren et al., 2014). Indeed, understanding the role of non-experts in ML is increasingly important as ML become more ubiquitous and affects the future of work (Frank et al., 2019). Non-experts have long been able to participate in data collection to train predictive models and interactive machine learning allows non-experts and ML to work together to better address pre-identified problems (Fails and Olsen Jr, 2003; Cheng and Bernstein, 2015; Crandall et al., 2018). However, as remarked by Yang et al. (2018), despite extensive research in these areas, little work has investigated how non-experts can take creative or editorial control to design their own applications of ML.

Crowdsourcing has long been used as an avenue to gather training data for machine learning methods (Lease, 2011). In this setting, it is important to understand the quality of worker responses, to prevent gathering bad data and to maximize the wisdom-of-the-crowd effects without introducing bias (Hsueh et al., 2009). Researchers have also studied active learning in this context, where an ML method is coupled in a feedback loop with responses from the crowd. One example is the Galaxy Zoo project (Kamar et al., 2012), where crowd workers are asked to classify photographs of galaxies while learning algorithms try to predict the galaxy classes and also manage quality by predicting the responses of individual workers.

While most crowdsourcing applications focus on relatively rote work such as basic image classification (Schenk and Guittard, 2009), many researchers have studied how to incorporate crowdsourcing into creative work. Some examples include the studies of Bernstein et al. (2015) and Teevan et al. (2016), both of which leverage crowd workers for prose writing; Kittur (2010), where the crowd helps with translation of poetry; Chilton et al. (2013), where the crowd develops taxonomic hierarchies; and Dontcheva et al. (2011), where crowd workers were asked to ideate new and interesting applications or uses of common everyday objects, such as coins. In the specific context of machine learning, Kaggle provides a competition platform for expert crowd participants to create predictive models, allowing data holders to crowdsource predictive modeling, but prediction tasks are still designed by the data providers not the crowd.

In many crowdsourcing applications where workers contribute novel information, a propose-and-rank algorithm is typically used to ensure that crowd resources are focused on high-quality contributions by ranking those contributions in advance (Siangliulue et al., 2015; Salganik and Levy, 2015). For example, the "Wiki Surveys" project (Salganik and Levy, 2015) asks volunteers to contribute and vote on new ideas 
for improving quality-of-life in New York City. Wiki Surveys couples a proposal phase with a ranking and selection step, to create ideas and then filter and select the best ideas for the city government to consider. None of these studies applied crowdsourced creativity to problems of machine learning or data collection, however.

Two sets of studies are perhaps most closely related to the research here. The first focuses on nonexperts who built ML tools that enable non-experts to build ML models, and investigated their goals, methods, and the challenges they encountered Yang et al. (2018). Yang et al. performed an empirical study, conducting interviews and surveys of non-experts, and their study serves as an important complement to our work. To the best of our knowledge, our study here is the first experimental consideration of non-experts and their ability to design prediction tasks.

The second set of studies all consider the non-expert design of predictive features, i.e. crowdsourced feature engineering (Bongard et al., 2013; Bevelander et al., 2014; Swain et al., 2015; Wagy et al., 2017). This work studies the crowdsourcing of survey questions in multiple problem domains. Participants answered questions related to a quantity of interest to the crowdsourcer, such as how much they exercised vs. their obesity level or how much laundry they did at home compared with their home energy usage. Those participants were also offered the chance to propose new questions related to the quantity of interest (obesity level or home energy use). Algorithms were deployed while crowdsourcing occurred to relate answers to proposed questions (the features) to the quantity of interest, and thus participants were performing crowdsourced feature engineering with the goal of contributing novel predictive features of interest. Another similar study, Flock (Cheng and Bernstein, 2015), demonstrates that features built by non-experts working together with algorithms can improve supervised classifiers. However, these studies all limit themselves to feature engineering, and still require experts to design the supervised learning prediction task itself, i.e. experts decide what is the quantity of interest to be predicted. Yet non-experts will be unable to apply ML to a new area of their own interest if they can only contribute features to pre-existing problems. Therefore, our work here generalizes this to ask individuals to design the entire prediction task, not just the features, by allowing non-experts to propose not only questions related to a quantity of interest, but also the quantity of interest itself.

\section{METHODS}

Here, we describe our procedures for non-experts to design prediction tasks which are then ranked, categorized, and data are collected for top-ranked prediction tasks. After data are collected for a given task, supervised learning models are trained and assessed with cross-validation to estimate the learnability of the prediction task. The University of Vermont Institutional Review Board granted Ethical approval to carry out the study (determination number CHRBSS: 15-039). Collected data are available on Figshare (https://doi.org/10.6084/m9.figshare.9468512). Crowdsourcing experiment details, including sample sizes and rewards, are given in Sec. 4.1.

\subsection{Prediction task ideation}

To understand how non-experts can design and use machine learning, we introduce a protocol for the creation and data collection of supervised learning prediction tasks (several example prediction tasks are shown in Table 1). Inspired by "propose-and-rank" crowd ideation methods (Sec. 2), the protocol proceeds in three phases: (i) prediction task proposal, (ii) task selection by ranking, and (iii) data collection for selected tasks. Proposed prediction tasks may also be categorized or labeled by workers, allowing us to understand properties of proposed tasks. This is an end-to-end procedure in that crowd workers generate all prediction tasks and data without manual interventions from the crowdsourcer, allowing us to understand what types of topics non-expert workers tend to be interested in, and whether machine learning models can be trained on collected data to make accurate predictions.

\subsubsection{Prediction task proposal}

In the first phase, a small number of workers are directed to propose sets of questions (see supplemental materials for the exact wording of these and all instructions we used in our experiments). Workers are instructed to provide a prediction task consisting of one target question and $p=4$ input questions. We focused on four input questions here to keep the proposal problem short; we discuss generalizing this in Sec. 5. Several examples of tasks proposed by workers are shown in Table 1. Workers are told that our goal is to predict what a person's answer will be to the target question after only receiving answers to 
Table 1. Examples of non-expert-proposed prediction tasks. Each task is a set of questions, one target and $p$ inputs, all generated entirely by non-experts (Sec. 3.1). After crowdsourced ranking (Sec. 3.1.2) and data collection (Sec. 3.1.3), answers to input questions form the data matrix $\mathbf{X}$ and answers to the target question form the target vector $y$. Machine learning algorithms try to predict the value of the target given only responses to the inputs. The learnability of the prediction task can then be assessed from the performance of these predictions. Prior work on crowdsourced feature engineering asks workers to contribute new predictive features (as input questions, in this case) for an expert-defined target. Here we ask workers to propose the entire prediction task not just the features.

\begin{tabular}{ll}
\hline & Prediction task \\
\hline Target: & What is your annual income? \\
Input: & You have a job? \\
Input: & How much do you make per hour? \\
Input: & How many hours do you work per week? \\
Input: & How many weeks per year do you work? \\
\hline Target: & Do you have a good doctor? \\
Input: & How many times have you had a physical in the last year? \\
Input: & How many times have you gone to the doctor in the past year? \\
Input: & How much do you weigh? \\
Input: & Do you have high blood pressure? \\
\hline Target: & Has racial profiling in America gone too far? \\
Input: & Do you feel authorities should use race when determining who to give scrutiny to? \\
Input: & How many times have you been racially profiled? \\
Input: & Should laws be created to limit the use of racial profiling? \\
Input: & How many close friends of a race other than yourself do you have? \\
\hline
\end{tabular}

the input questions. Describing the prediction task proposal problem in this manner allows workers to envision the underlying goal of the supervised learning task without the need to discuss data matrices, response variables, predictors, or other field-specific vocabulary. Workers were also instructed to use their judgment and experience to determine "interesting and important" tasks. Importantly, no examples of questions were shown to workers, to help ensure they were not biased in favor of the example (we investigate this bias with a randomized trial; see Secs. 3.3 and 4.4). Workers were asked to write their questions into provided text fields, ending each with a question mark. They were also asked to categorize the type of answer expected for each question; for simplicity, we directed workers to provide questions whose answers were either numeric or true/false (Boolean), though this can be readily generalized. Lastly, workers in the first phase are also asked to provide answers to their own questions.

\subsubsection{Prediction task ranking and selection}

In the second phase, new workers are shown previously proposed tasks, along with instructions again describing the goal of predicting the target answer given the input answers, but these workers are asked to (i) rank the task according to our criteria (described below) but using their own judgment, and (ii) answer survey questions describing the tasks they were shown. It is useful to keep individual crowdsourcing tasks short, so it is generally too burdensome to ask each worker to rank all $N$ tasks. Instead, we suppose that workers will study either one task or a pair of tasks depending on the ranking procedure, complete the survey questions for the task(s), and, if shown a pair of tasks, to rate which of the two tasks they believed was "better" according to the instructions. To use these ratings to develop a global ranking of tasks from "best" to "worst", we apply top- $K$ ranking algorithms (Sec. 3.2). These algorithms select the $K$ most suitable tasks to pass along to phase three.

Task categorization As an optional part of phase two, data can be gathered to categorize what types of tasks are being proposed, and what are the properties of those tasks. To categorize tasks, we asked workers what the topic of each task is, whether questions in the task were subjective or objective, how well answers to the input questions would help to predict the answer to the target question, and what kind of responses other people would give to some questions. We describe the properties of proposed tasks in 
Sec. 4.

\subsubsection{Data collection and supervised learning to assess learnability}

In phase three, workers were directed to answer the input and target questions for the tasks selected during the ranking phase. Workers could answer the questions in each selected task only once but could work on multiple prediction tasks. In our case, we collected data from workers until each task had responses from a fixed number of unique workers $n$, but one could specify other criteria for collecting data. The answers workers give in this phase create the datasets to be used for learning. Specifically, the $n \times p$ matrix $\mathbf{X}$ consists of the $n$ worker responses to the $p$ input questions (we can also represent the answers to each input question $i$ as a predictor vector $x_{i}$, with $\mathbf{X}=\left[x_{1}, \ldots, x_{p}\right]$ ). Likewise, the answers to the target question provide the supervising or target vector $y$.

After data collection, supervised learning methods can be applied to find the best predictive model $\hat{f}$ that relates $y$ and $\mathbf{X}$, i.e., $y=\hat{f}(\mathbf{X})$. Then learnability of the prediction task and data can be assessed by model performance using cross-validation, although this assessment depends on the predictive model used, and more advanced models may lead to improved learnability. In our case, we focused on random forests (Breiman, 2001) as our predictive model, a commonly used and general-purpose ensemble learning method. Random forests work well on both linear and nonlinear prediction tasks and can be used for both regressions (where $y$ is numeric) and classifications (where $y$ is categorical). However, any supervised learning method can be applied in this context. For hyperparameters used to fit the forests, we chose 200 trees per forest, a split criterion of MSE for regression and Gini impurity for classification, and tree nodes are expanded until all leaves are pure or contain fewer than 2 samples. These are commonly accepted choices for hyperparameters, but of course careful tuning of these values (using appropriate cross-validation) can only result in better learning than we report here.

\subsection{Ranking proposed prediction tasks}

Not all workers will propose meaningful tasks, so it is important to include a ranking step (phase two) that filters out low-quality (less meaningful) tasks while promoting high-quality (more meaningful) tasks.

To ground our ranking process, here we define a prediction task as "meaningful" if it is both important, as determined by crowd judgments and learnable, as assessed by cross-validation of a trained predictive model. A non-important prediction task may be one that leads to unimportant or unimpactful broader consequences, the target variable may not be worth predicting, or the task may simply recapitulate known relationships ("Do you think $1+1=2$ ?"). As importance can be subjective, here we rely on the crowd to collectively certify whether a task is important or not according to their own criteria. Although it may be necessary to guide non-experts to specific areas of interest (see discussion), here we avoid introducing specific judgments or criteria so that we can see what "important" prediction tasks are proposed by non-experts.

To be meaningful as a prediction task, the task must also be learnable. Indeed, another characteristic of poor prediction tasks is a lack of learnability, defined as the ability for a predictive model trained on data collected for the prediction task to accurately generalize to unseen data. For a binary classification task, for example, one symptom of poor learnability (but not the only issue) is an imbalance of the class labels. For example, the target question "Do you think running and having a good diet are healthy?" is likely to lead to very many "true" responses and very few "false" responses. Such data lacks diversity (in this case, in the labels), which makes learning difficult. Of course, while a predictive model in such a scenario is not especially useful, the relationships and content of the target and input questions are likely to be meaningful, as we saw in some of our examples; see supplemental materials). In other words, a predictive task can be about an important topic or contain important information, but if it is not learnable then it is not meaningful as a prediction task.

Here we detail how to use crowd feedback to efficiently rank tasks based on importance and learnability. We refer to the ratings given by the crowd as "perceived importance" and "perceived learnability" the reflect the subjective nature of their judgments. The outcome of this ranking (Sec. 4) also informs our investigation of RQ4. In the context of crowdsourcing prediction tasks, the choice of ranking criteria gives the crowdsourcer flexibility to guide workers in favor of, not necessarily specific types of tasks, but tasks that possess certain features or properties. This balances the needs of the crowdsourcer (and possible budget constraints) without restricting the free-form creative ideation of the crowd. 


\subsubsection{Perceived importance ranking}

We asked workers to use their judgment to estimate the "importance" of tasks (see supplemental materials for the exact wording of instructions). To keep workloads manageable, we ask workers to compare two tasks at a time, with a simple "Which of these two tasks is more important?"-style question. This reduces the worker's assignment to a pairwise comparison. Yet, even reduced to pairwise comparisons, the global ranking problem is still challenging, as one needs $\mathscr{O}\left(N^{2}\right)$ pairwise comparisons for $N$ tasks, comparing every task to every other task. Furthermore, importance is generally subjective, so we need the responses of many workers and cannot rely on a single response to a given pair of tasks. Assuming we require $L$ independent worker comparisons per pair, the number of worker responses required for task ranking grows as $\mathscr{O}\left(L N^{2}\right)$.

Thankfully, ranking algorithms can reduce this complexity. Instead of comparing all pairs of tasks, these algorithms allow us to compare a subset of pairs to infer a latent score for each task, then rank all tasks according to these latent scores. For this work, we chose the following top- $K$ spectral ranking algorithm, due to Negahban et al. (2017), to rank non-expert-proposed tasks and extract the $K$ best tasks for subsequent crowdsourced data collection. The algorithm uses a comparison graph $G=(V, E)$, where the $N$ vertices denote the tasks to be compared, and comparison between two tasks $i$ and $j$ occurs only if $(i, j) \in E$. For our specific crowdsourcing experiment, we solicited $N=50$ tasks during the proposal phase, so here we generated a single Erdős-Rényi comparison graph of 50 nodes with each potential edge exists independently with probability $p=1.5 \log (N) / N$ (this $p$ ensures $G$ is connected), and opted for $L=15$. Increasing $L$ can improve ranking accuracy, but doing so comes at the cost of more worker time and crowdsourcer resources. The choice of an Erdős-Rényi comparison graph here is useful: when all possible edges are equally and independently probable, the number of samples needed to produce a consistent ranking is nearly optimal (Negahban et al., 2017).

\subsubsection{Perceived learnability ranking}

As discussed above, prediction tasks lack learnability when (among other reasons) there is insufficient diversity in the dataset. If nearly every observation is identical, there is not enough "spread" of data for the supervised learning method to train upon; no trends will appear if every response to the input questions is identical or if every value of the target variable is equal. To avoid collecting data for tasks that are not learnable, and to assess how well non-experts can make determinations about learnability, we seek a means for workers to estimate for us the learnability of a proposed task when shown the input and target questions. The challenge is providing workers with an assignment that is sufficiently simple for them to perform quickly yet does not require training or background in how supervised learning works.

To address this challenge, we designed an assignment to ask workers about their opinions of the set of answers we would receive to a given question (a form of meta-knowledge). We focused on a lack of diversity in the target variable. We also limited ourselves to Boolean (true/false) target questions, although it is straightforward to generalize to regressions (numeric target questions) by rephrasing the assignment slightly. Specifically, we asked workers what proportion of respondents would answer "true" to the given question. Workers gave a 1-5 Likert-scale response from (1) "No one will answer true" to (3) "About half will answer true" to (5) "Everyone will answer true". The idea is that, since a diversity of responses is generally necessary (but not sufficient) for (binary) learnability, classifications that are balanced between two class labels are more likely to be learnable. To select tasks, we use a simple ranking procedure to seek questions with responses predominantly in the middle of the Likert scale. Specifically, if $t_{i j} \in\{1, \ldots, 5\}$ is the response of the $i$-th worker to prediction task $j$, we take the aggregate learnability ranking to be

$$
t_{j}=\left|3-\frac{\sum_{i=1}^{W} t_{i j} \delta_{i j}}{\sum_{i=1}^{W} \delta_{i j}}\right|
$$

where $W$ is the total number of workers participating in learnability ranking tasks, and $\delta_{i j}=1$ if worker $i$ ranked task $j$, and zero otherwise. The closer a task's score is to 3 , the more the workers agree that target answers would be evenly split between true and false, and so we rank tasks based on the absolute deviation from the middle score of 3. While Eq. (1) is specific to a 1-5 Likert scale variable, similar scores can be constructed for any ordinal variable.

This perceived learnability ranking task can be combined with a pairwise comparison methodology like the one described for perceived importance ranking. In our case, we elected to perform a simpler onetask assignment because perceived learnability ranking from Eq. (1) only requires examining the target 
question and because workers are less likely to need a relative baseline here as much as they may with perceived importance ranking, where a contrast effect between two tasks is useful for judging subjective values such as importance. Due to time and budget constraints we also took $K=5$ for experiments using this ranking phase.

There are limitations of this approach to perceived learnability beyond focusing only on classifications. For one, soliciting this information does not address a potential lack of diversity in the $\mathbf{X}$ data, nor do we consider the perceived learnability of regressions. Likewise, it does not address myriad other issues that affect learnability, such as the capacity of the predictive model (Vapnik, 2013; Valiant, 1984). See Sec. 5 for further discussion.

\subsection{Randomized trial for assignment design: giving examples}

In addition to the crowdsourced proposal, ranking and data collection, we augmented our study with a randomized trial investigating the design of the prediction task proposal assignment (RQ3). Specifically, we investigated the role of providing an example of a prediction task.

Care must be taken when instructing workers to propose prediction tasks. Without experience in machine learning, they may be unable to follow instructions which are too vague or too reliant on machine learning terminology. Providing an example with the instructions is one way to make the assignment more clear while avoiding jargon. An example helps avoid the ambiguity effect (Ellsberg, 1961), where workers are more likely to avoid the task because they do not understand it. However, there are potential downsides as well: introducing an example may lead to anchoring (Tversky and Kahneman, 1974) where workers will be biased towards proposing tasks related to the example and may not think of important, but different prediction tasks.

Workers who did not participate in previous assignments were randomly assigned to one of three comparison groups or "arms" when accepting the prediction task proposal assignment (simple random assignment). One arm had no example given with the instructions and was identical to the assignment studied previously (Sec. 4.2). This arm serves as a baseline or control group. The second arm included with the instructions an example related to obesity (An example target question is: "Are you obese?"), and the third arm presented an example related to personal finance (An example target question is: "What is your current life savings?"). The presence or absence of an example is the only difference across arms; all other instructions were identical and, crucially, workers were not instructed to propose prediction tasks related to any specific topical domain or area of interest.

After we collected new prediction tasks proposed by workers who participated in this randomized trial, we then initiated a followup task categorization assignment (Sec. 3.1.2) identical to the categorization assignment discussed previously but with two exceptions: we asked workers to only look at one prediction task per assignment and we did not use a comparison graph as here we will not rank these tasks for subsequent data collection. The results of this categorization assignment allow us to investigate the categories and features of the proposed prediction tasks and to see whether or not the tasks differ across the three experimental arms.

\section{RESULTS}

\subsection{Crowdsourcing assignments}

We performed our experiments using Amazon Mechanical Turk during August 2017. Assignments were performed in sequence, first prediction task proposal (phase one), then ranking and categorization (phase two), then data collection (phase three). These assignments and the numbers of responses and numbers of workers involved in each are detailed in Table 2, as are the rewards workers were given. Rewards were determined based on estimates of the difficulty or time spent on the assignment, so proposing a prediction task had a much higher reward (\$3 USD) than providing data by answering the task's questions ( $\$ 0.12$ USD). No responses were filtered out at any point, although a small number of responses (less than 1\%) were not successfully recorded.

We solicited $N=50$ prediction tasks in phase one, compensating Mechanical Turk workers $\$ 3$ for the assignment. Workers could submit only one task. A screenshot of the assignment interface for this phase (and all phases) is shown in the supplemental materials. Some example prediction tasks provided by crowd workers are shown in Table 1; all 50 tasks are shown in the supplemental materials. After these tasks were collected, phase two began where workers were asked to rate the tasks by their perceived importance and perceived learnability and to categorize the properties of the proposed tasks. Workers 


\begin{tabular}{rlcrr}
\hline Phase & Assignment & Reward & Responses & Workers \\
\hline 1 & Prediction task proposal & $\$ 3.00$ & 50 & 50 \\
2 & Perceived importance rating \& task categorization & $\$ 0.25$ & 2042 & 239 \\
2 & Perceived learnability rating & $\$ 0.05$ & 835 & 83 \\
3 & Data collection, perceived importance tasks & $\$ 0.12$ & 2004 & 495 \\
3 & Data collection, perceived learnability tasks & $\$ 0.12$ & 990 & 281 \\
\hline 1 & Prediction task proposal (randomized trial) & $\$ 3.00$ & 90 & 90 \\
2 & Task categorization (randomized trial) & $\$ 0.13$ & 458 & 61 \\
\hline
\end{tabular}

Table 2. Summary of crowdsourcing assignments. Rewards in USD.

were compensated $\$ 0.25$ per assignment in phase two and were limited to examining at most 25 tasks total. After the second phase completed, we chose the top-10 most perceived important tasks and the top-5 most perceived learnable tasks (Sec. 3.2) to pass on to data collection (phase three). We collected data for these tasks until $n=200$ responses were gathered for each prediction task (we have slightly less responses for some tasks as a few responses were not recorded successfully; no worker responses were rejected). Workers in this phase could respond to more than one task but only once to each task.

For the randomized trial on the effects of providing an example prediction task (Sec. 3.3), we collected $N=90$ proposed prediction tasks across all three arms (27 in the no-example baseline arm, 33 in the obesity example arm, and 30 in the savings example arm), paying workers as before. We then collected 458 task categorization ratings, gathering ratings from 5 or more distinct workers per proposed task (no worker could rate more than 25 different prediction tasks). Since only one task was categorized per assignment instead of two, workers were paid $\$ 0.13$ per assignment instead of the original $\$ 0.25$ per assignment.

\subsection{Characteristics of proposed tasks}

We examined the properties of prediction tasks proposed by workers in phase one (RQ2). We measured the prevalence of Boolean and numeric questions. In general, workers were significantly in favor of proposing Boolean questions over numeric questions. Of the $N=50$ proposed tasks, 34 were classifications (Boolean target question) and 16 were regressions (numeric target question). Further, of the 250 total questions provided across the $N=50$ tasks, $177(70.8 \%)$ were Boolean and 73 were numeric $(95 \% \mathrm{CI}$ on the proportion of Boolean: $64.74 \%$ to $76.36 \%$ ), indicating that workers were significantly in favor of Boolean questions over numeric. Likewise, we also found an association between whether the input questions were numeric or Boolean given the target question was numeric or Boolean. Specifically, we found that prediction tasks with a Boolean target question had on average 3.12 Boolean input questions out of 4 (median of 4 Boolean input questions), whereas tasks with a numeric target question had 2.31 Boolean input questions on average (median of 2 Boolean input questions). The difference was significant (Mann-Whitney test: $U=368.5, n_{\text {bool }}=34, n_{\text {num }}=16, p<0.02$ ). Although it is difficult to draw a strong conclusion from this test given the amount of data we have (only $N=50$ proposed prediction tasks), the evidence we have indicates that workers tend to think of the same type of question for both the target and the inputs, despite the potential power of mixing the two types of questions.

To understand more properties of the questions workers proposed, we asked workers to categorize prediction tasks by giving survey questions about the tasks as part of the perceived importance rating assignment. We used survey questions about the topical nature or domain of the task (Fig. 1), whether the inputs were useful at predicting the target (Fig. 2), and whether the questions were objective or subjective (Fig. 3). Prediction task categories (Fig. 1) were selected from a multiple choice categorization we determined manually. Tasks about demographic or personal attributes were common, as were political and current events. Workers generally reported that the inputs were useful at predicting the target, either rating "agree" or "strongly agree" to that statement (Fig. 2). Many types of tasks were mixes between objective and subjective questions, while tasks categorized as "factual" tended to contain the most objective questions and tasks categorized as "other/unsure" contained the most subjective questions, indicating a degree of meaningful consistency across the categorization survey questions.

When ranking classification tasks by perceived learnability, we asked workers about the diversity of responses they expected others to give to the Boolean target question, whether they believed most people 

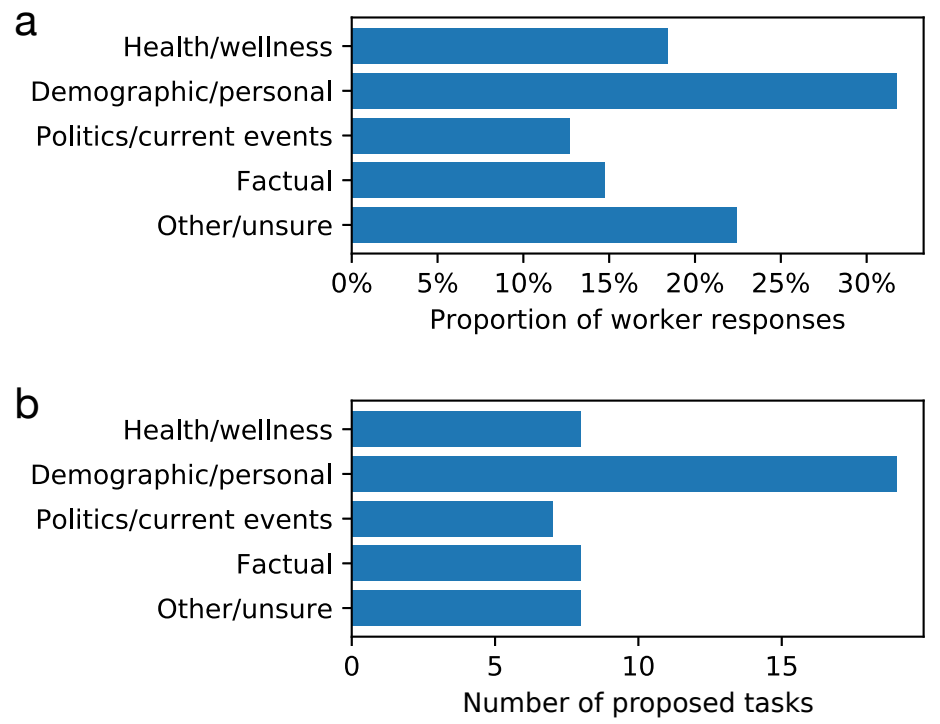

Figure 1. Topical categories of proposed prediction tasks. Panel (b) counts the majority categorization of each task.

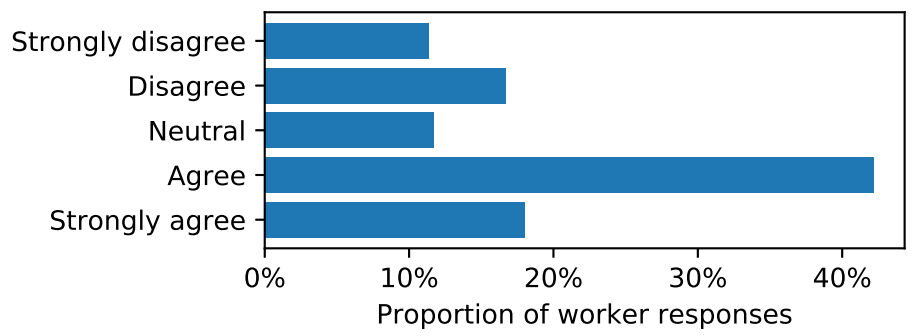

Figure 2. Worker responses to, "Are the input questions useful at predicting answers to the target question?" when asked to categorize proposed prediction tasks.

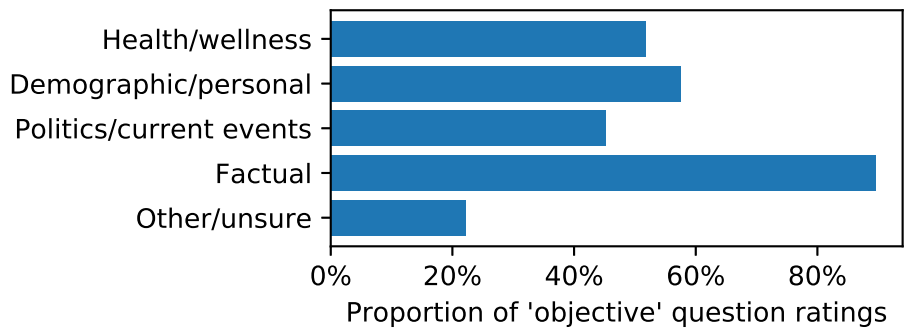

Figure 3. Proportion of question ratings of 'objective' instead of 'subjective' vs. the majority category of the prediction task. 


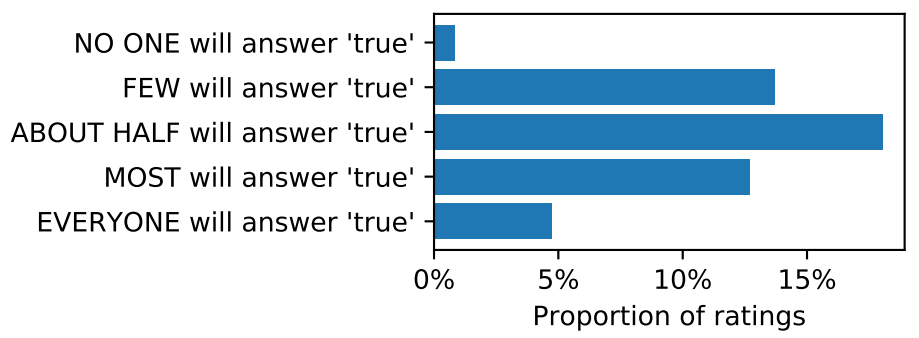

Figure 4. Categorized diversity of the (Boolean) target questions.

would answer false to the target question, or answer true, or if people would be evenly split between true and false (Fig. 4). We found that generally there was a bias in favor of positive (true) responses to the target questions, but that workers felt that many questions would have responses to the target questions be split between true and false. This bias is potentially important for a crowdsourcer to consider when designing her own tasks, but seeing that most Boolean target questions are well split between true and false response also supports that workers are proposing useful tasks; if the answer to the target question is always false, for example, then the input questions are likely not necessary, and the workers generally realize this when proposing their tasks.

\subsection{Prediction task learnability: supervised learning on collected data}

Given the proposed prediction tasks and the selection of tasks for subsequent data collection, it is also important to quantify predictive model performance on these tasks (RQ5), allowing us to determine if the tasks are learnable given the data and model used. Since workers are typically not familiar with supervised learning, there is a risk they may be unable to propose learnable prediction tasks. At the same time, however, workers may not be locked into traditional modes of thinking, such as assumptions that predictors are linearly related to the target, leading to interesting and potentially unexpected combinations of predictor variables and the response variable.

Here we trained and evaluated random forest regressors and classifiers (Sec. 3.1.3), depending on whether the proposer flagged the target question as either numeric or Boolean, using the data collected for the 15 selected prediction tasks. Learnability (predictive performance) was measured using the coefficient of determination for regressions and mean accuracy for classifications, as assessed with k-fold cross-validation (stratified k-fold cross-validation if the task is a classification). To assess the variability of performance over different datasets, we used bootstrap replicates of the original crowd-collected data to estimate a distribution of cross-validation scores. There is also a risk that class imbalance may artificially inflate performance: when nearly every target variable is equal always predicting the majority class label can appear to perform well. To account for class imbalance, we also trained on a shuffled version of each task's dataset, where we randomly permuted the rows of the data matrix $\mathbf{X}$, breaking the connection with the target variable $y$. If models trained on these data performed similarly to models trained on the real data, then it is difficult to conclude that learning has occurred, although this does not mean the questions are not meaningful, only that the data collected does not lead to useful predictive models.

The results of this learnability assessment procedure are shown in Fig. 5. We quantify the practical effect size with Cohen's $d$ comparing the real training data to the shuffled control, and in Fig. 5 we highlight in green any tasks with Cohen's $d>2$. Many of the 10 perceived importance-ranked tasks in Figs. 5(a-j) demonstrate a lack of learnability but at least two of the ten perceived important tasks, one regression and one classification, also show significant learning ${ }^{1}$. At the same time, four out of the five perceived learnability-ranked tasks (Figs. 5(k-0)) showed strong predictive performance, further indicating the ability of non-experts to perform learnability assessments.

These results show that, while many of the worker-proposed prediction tasks are difficult to learn on, and caution must be taken to instruct non-experts about the issue of class imbalance, it is possible to generate tasks where learning can be successful and to assess this with an automatic procedure such as testing the differences of the distributions shown in Fig. 5. Further, for the classification tasks in Figs. $5 \mathrm{k}-\mathrm{o}$, by assessing learnability (predictive performance) using cross-validation, we can compare

${ }^{1}$ One regression showed poor performance scores for reasons we detail in the discussion. 

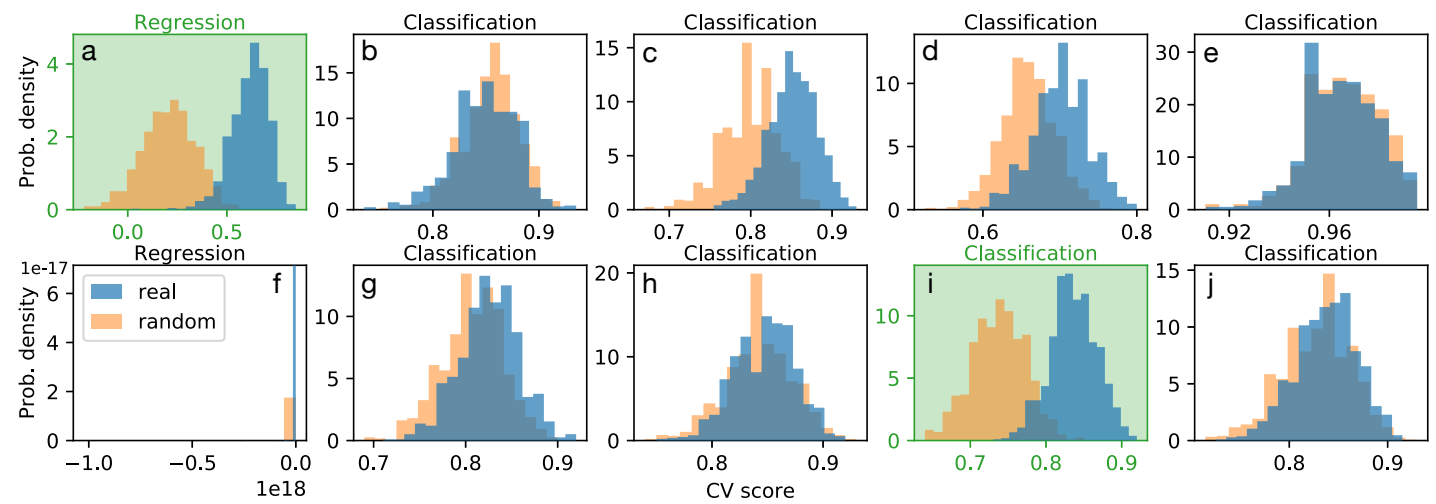

(a-j) Top perceived importance problems

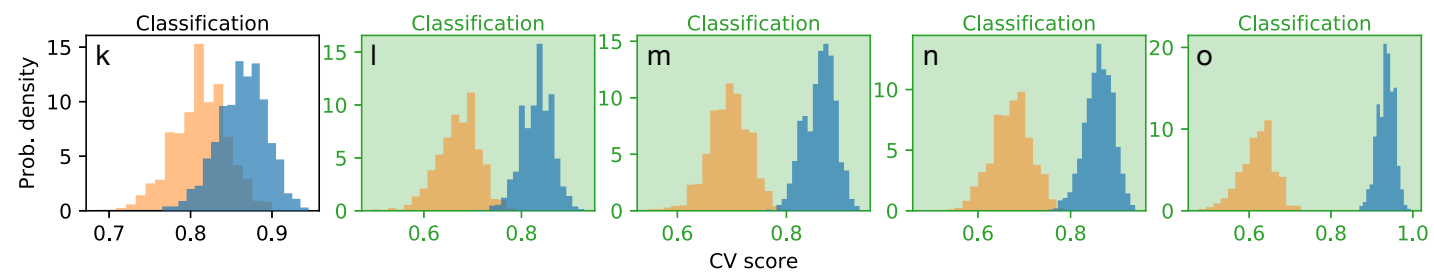

(k-o) Top perceived learnability (classification) problems

Figure 5. Cross-validation scores for (a-j) the top-10 perceived importance ranked prediction tasks (Sec. 3.2.1) and ( $\mathrm{k}-\mathrm{o}$ ) the top-5 perceived learnable prediction tasks (Sec. 3.2.2). Performance variability was assessed with bootstrap replicates of the crowdsourced datasets and class imbalance was assessed by randomizing the target variable relative to the input data. At least two of the perceived importance tasks and four of the perceived learnable tasks - highlighted in green - demonstrate significant learnability (predictive performance) over random (Cohen's $d>2$ ). Note that the regression task in panel (f) showed poor predictive performance for reasons we describe in the discussion.

with non-expert judgments of perceived learnability. Despite perceived learnability being oversimplified (only considering the issue of class imbalance), we found that most perceived learnable tasks demonstrated learnability in practice.

\subsection{Randomized trial: giving examples of prediction tasks}

To understand what role an example may play-positive or negative-in task proposal, we conducted a randomized trial investigating the instructions given to the workers. As described in Sec. 3.3, we conducted a three-armed randomized trial. Workers who did not participate in the previous study were asked to propose a prediction task with instructions that either contained no example (baseline arm), contained an example related to obesity (obesity arm), or contained an example related to personal savings (savings arm). The prediction tasks proposed by members of these arms were categorized and rated and from these ratings we study changes in task category, usefulness of input questions at answering the target question, if the questions are answerable or unanswerable, and if the questions are objective or subjective, as judged by workers participating in the followup rating tasks.

The results of this trial are summarized in Fig. 6 and Tables 3 and 4. We found that:

- Prediction task categories changed due to the examples (Fig. 6), with more 'demographic/personal' tasks, fewer 'politics/current events', and fewer 'factual' questions under the example treatments compared with the baseline. This change was significant $\left(\chi^{2}=52.73, p<0.001\right)$.

- Workers shown the savings example were significantly more likely than workers in other arms to propose questions with numeric responses instead of Boolean responses: $60 \%$ of questions proposed in the savings arm were numeric compared with $25 \%$ in the no-example baseline (Fisher exact, $p<0.001)$. 


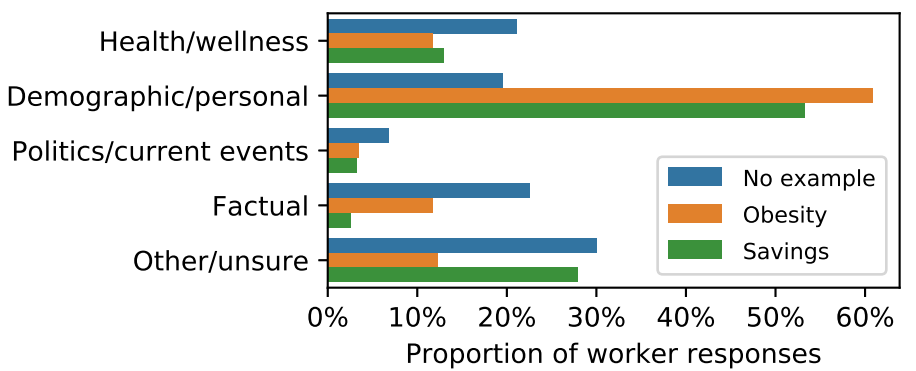

Figure 6. Categories of proposed prediction tasks under the different instructional treatments. Tasks proposed by workers who saw either example were more likely to be rated as demographic or personal and less likely to be considered factual. Interestingly, the obesity example led to fewer proposed tasks related to health or wellness.

\begin{tabular}{lccc}
\hline Rating or feature (variable type) & $\begin{array}{c}\text { Mean } x \\
\text { baseline }\end{array}$ & $\begin{array}{c}\text { Mean } x \\
\text { obesity }\end{array}$ & $\begin{array}{c}\text { Mean } x \\
\text { savings }\end{array}$ \\
\hline Perceived task importance $(x=1-5$ Likert; 5: Strongly agree) & 3.09 & 3.16 & $\mathbf{3 . 5 0}$ \\
Inputs are useful $(x=1-5$ Likert; 5: Strongly agree) & 3.36 & $\mathbf{3 . 9 1}$ & 3.67 \\
Questions are answerable $(x=1)$ or unanswerable $(x=0)$ & 0.82 & $\mathbf{0 . 9 2}$ & $\mathbf{0 . 9 2}$ \\
Questions are objective $(x=1)$ or subjective $(x=0)$ & 0.59 & 0.67 & 0.68 \\
Questions are numeric $(x=1)$ or Boolean $(x=0)$ & 0.25 & 0.35 & $\mathbf{0 . 6 0}$ \\
\hline
\end{tabular}

Table 3. Typical ratings and features of prediction tasks proposed under the three instruction types (the no-example baseline, the obesity example, and the savings example). Bold treatment quantities show a significant difference $(p<0.05)$ from the baseline (Chi-square tests for Likert $x$; Fisher exact tests for $\operatorname{binary} x$ ).

\begin{tabular}{lllllll}
\hline & & \multicolumn{2}{c}{ Baseline vs. obesity } & & \multicolumn{2}{c}{ Baseline vs. savings } \\
Difference in & Test & statistic & p-value & & statistic & p-value \\
\hline task categories (see Fig. 6) & Chi-square & $52.73 *$ & $<10^{-10}$ & & $52.73 *$ & $<10^{-9}$ \\
perceived task importance (see Table 3) & Chi-square & 8.57 & $>0.05$ & & $11.84 *$ & $<0.02$ \\
inputs are useful (see Table 3) & Chi-square & $16.35 *$ & $<0.005$ & 7.20 & $>0.1$ \\
answerable/unanswerable (see Table 3) & Fisher exact & $-*$ & 0.0083 & & $-*$ & 0.012 \\
objective/subjective (see Table 3) & Fisher exact & - & 0.12 & & 0.078 \\
numeric/Boolean (see Table 3) & Fisher exact & $-*$ & 0.041 & $-*$ & $<10^{-8}$ \\
\hline
\end{tabular}

Table 4. Statistical tests comparing the categories and ratings given for prediction tasks generated under the no-example baseline with the categories and ratings of tasks generated under the obesity example and savings example baselines. For categorical and Likert-scale ratings we used a Chi-square test of independence while for binary ratings we used a Fisher exact test. Significant results $(p<0.05)$ are denoted with $*$. 
- All three arms were rated as having mostly answerable questions, with a higher proportion of answerable questions for both example treatments: $92 \%$ of ratings were 'answerable' for both example treatments compared with $82 \%$ for the baseline (Table 3). Proportions for both example treatments were significantly different from the baseline (Fisher exact, $p<0.02$ ).

- Workers more strongly agreed that the inputs were useful at predicting the target for prediction tasks proposed by workers under the example treatments than the tasks proposed under the no-example baseline. The overall increase was not large, however, and tested as significant $\left(\chi^{2}=16.35, p<\right.$ 0.005 ) only for the savings example vs. the baseline.

- Questions proposed under the example treatments were more likely to be rated as objective than questions proposed under the no-example baseline: $67 \%$ and $68 \%$ of ratings were 'objective' for the obesity and savings examples, respectively, compared with 59\% for the baseline (Table 3). However, this difference was not significant for either treatment (Fisher exact, $p>0.05$ ).

Taken together, the results of this experiment demonstrate that examples, while helping to explain the assignment, will lead to significant changes in the features and content of proposed prediction tasks. Individuals may provide better and somewhat more specific questions, but care may be needed when selecting which examples to use, as individuals may potentially anchor onto those examples in some ways when designing their own prediction tasks. Such anchoring may be undesired but it may also be useful at "nudging" non-experts towards tasks related to a problem area of interest; see discussion for more.

\section{DISCUSSION}

Here we studied the abilities of non-experts to independently design supervised learning prediction tasks. Recruiting crowd workers as non-experts, we determined that non-experts were able to propose important or learnable prediction tasks with minimal instruction, but several challenges demonstrate that care should be taken when developing instructions as non-experts may propose trivial or "bad" tasks. Analyzing the proposed prediction tasks, we found that non-experts tended to favor Boolean questions over numeric questions, that input questions tended to be positively correlated with target questions, and that many prediction tasks were related to demographic or personal attributes.

Learnability is estimated using cross-validation (Fig. 5). This estimate encompasses the prediction task, the predictive model, and the training data. However, we also ask non-experts to estimate learnability. Can non-experts do this without knowing about cross-validation? Our answer is yes, for the case of classification. Despite class imbalance, our measure of perceived learnability, being only one factor affecting learnability, four out of five of the top-rated "learnable" tasks demonstrated learnability using cross-validation. Meanwhile, two of the ten top-rated "important" tasks also demonstrated learnability, indicating that non-experts can generate meaningful prediction tasks.

It is worth speculating on the origins of these results. For instance, the crowd workers could favor Boolean questions because they can be proposed more quickly (due to being less cognitively demanding) and crowd workers wish to work quickly to maximize their earnings. Or they could favor Boolean questions because their prior experience leaves them less familiar with numerical quantities. Likewise, a focus on demographic or personal attributes within prediction tasks could reflect the inherent interests of the participants or could be due to influences from prior work on the crowdsourcing platform. More generally, a useful future direction of study is to better understand the backgrounds of the non-experts. For example, how is the prediction task associated with the prior experience, domain expertise or education level of the non-expert who proposed the task?

To better understand how framing the problem of designing a prediction task may affect the tasks workers proposed, we also conducted a randomized trial comparing tasks proposed by workers shown no example to those shown examples, and found that examples significantly altered the categories of proposed prediction tasks. These findings demonstrate the importance of carefully considering how to frame the assignment, but they also reveal opportunities. For example, it is less common for non-expert workers to mix Boolean and numeric questions, but workers that do propose such mixtures may be identified early on and then steered towards particular tasks, perhaps more difficult tasks. Likewise, given that examples have a powerful indirect effect on prediction task design, examples may be able to "nudge" non-experts in one direction while retaining more creativity than if the non-experts were explicitly restricted to designing a particular type of prediction task. We saw an example of this in Sec. 4.4: non-expert workers shown the 
savings example were over 2.5 times more likely to propose numeric questions than workers shown no example.

Our experiments have limitations that should be addressed in future work. For one, we only considered non-experts on the Mechanical Turk platform, and work remains to see how our results generalize beyond that group (Berinsky et al., 2012; Chandler et al., 2019). It is also important to explore more ML methods than we used here to learn predictive models on non-expert prediction tasks, especially as ML is a rapidly-changing field. Indeed, learnability can only be improved upon with more advanced methods. Further, assessing learnability only through judgments of class imbalance is an oversimplification of the issues that can affect learnability, although this oversimplification helped enable non-experts to judge learnability, at least in a limited manner, as shown in Fig. 5. Likewise, our ranking procedure considered the perceived importance and perceived learnability of prediction tasks separately, yet the most meaningful tasks should be ranked highly along both dimensions. With our prediction task proposal framework, we limited non-experts to numeric or Boolean questions, and a total of five questions per prediction task, but varying the numbers of questions and incorporating other answer types are worth exploring. For numeric questions, one important consideration is the choice of units. Indeed, we encountered one regression task (mentioned previously; see supplemental materials) where learning failed because the questions involved distances and volumes, but workers were not given information on units, leading to wildly varying answers. This teaches us that non-experts may need to be asked if units should be associated with the answers to numeric questions when they are asked to design a prediction task.

Our experiment procedures were used to address this study's research questions, but in the context of crowdsourcing, our end-to-end propose-and-rank procedure for soliciting prediction tasks can serve as an efficient crowdsourcing algorithm. To avoid wasting resources on low-quality or otherwise inappropriate prediction tasks, efficient task selection and data collection algorithms are needed to maximize the ability of a crowd of non-experts to collectively generate suitable prediction tasks while minimizing the resources required from a crowdsourcer. When allowing creative contributions from a crowd, a challenge is that workers may propose trivial or uninteresting tasks. This may happen intentionally, due to bad actors, or unintentionally, due to workers misunderstanding the goal of their assignment. Indeed, we encountered a number of such proposed prediction tasks in our experiments, further underscoring the need for both careful instructions and the task ranking phase. Yet, we found that the task ranking phase did a reasonable job at detecting and down-ranking such prediction tasks, although there is room to improve on this further, for example by reputation modeling of workers or implementing other quality control measures (Allahbakhsh et al., 2013; Scholer et al., 2011; Lease, 2011) or perhaps by providing dynamic feedback earlier, when tasks are being proposed. More generally, it may be worth combining the ranking and data collection phases, collecting data immediately for all or most prediction tasks but simultaneously monitoring the tasks as data are collected for certain specifications and then dynamically allocating more incoming workers to the most suitable subset of prediction tasks (Li et al., 2016; McAndrew et al., 2017).

Allowing the crowd to propose prediction tasks requires more work from the researcher on prediction task specification than in traditional applied machine learning. Traditionally, considerable effort is placed on model fitting, model validation, and feature engineering. AutoML will become increasingly helpful at model fitting and model validation while non-experts contributing predictive features may take some or even all of the feature engineering work off the researcher's hands. If crowd-proposed tasks are used, the researcher will need to consider how best to specify prediction task requirements. While here we allowed the crowd to ideate freely about tasks, with the goal of understanding what tasks they were most likely to propose, in practice a researcher is likely to instead focus on particular types of prediction tasks. For example, a team of medical researchers or a team working at an insurance firm may request only prediction tasks focused on health care. Future work will investigate methods for steering the crowd towards topics of interest such as health care, in particular on ways of focusing the crowd while biasing workers as little as possible.

Many interesting, general questions remain. For one, more investigation is needed into how nonexperts work on ML prediction tasks. Which component or step of designing a prediction task is most challenging for non-experts? What aspects of ML, if any, are most important to teach to non-experts? Can studies of non-experts help inform teaching methodologies for turning ML novices into experts? Likewise, can teaching methodologies for learning about ML inform better ways to help non-experts contribute to machine learning? 


\section{CONCLUSION}

In this study, we investigated how well non-experts, individuals without a background in machine learning, could contribute to machine learning. While non-experts have long contributed training data to power pre-defined machine learning tasks, it remains unclear whether and to what extent non-experts can apply existing ML methods in new problem areas. We asked non-experts to design their own supervised learning prediction tasks, then asked other non-experts to rank those tasks according to criteria of interest. Finally, training data were collected for top-ranked tasks and machine learning models were fit to those data. We were able to demonstrate that performant models can be trained automatically on non-expert tasks. We also studied the characteristics of proposed tasks, finding that many tasks were focused on health, wellness, demographics, or personal topics, that numeric questions were less common than Boolean questions, and that there was a mix of both subjective and objective questions, as rated by crowd workers. Using a randomized trial on the effects of instructional messages showed that simple examples caused non-experts to change their approaches to prediction tasks: for example, non-experts shown an example of a prediction task related to personal finance were significantly more likely to propose numeric questions.

In general, the more that non-experts can contribute creatively to machine learning, and not merely provide training data, the more we can leverage areas such as automatic machine learning to design new and meaningful applications of machine learning. More diverse groups can benefit from such applications, allowing for broader participation in jobs and industries that are changing due to machine-learning-driven workplace automation.

\section{ACKNOWLEDGMENTS}

Any opinions, findings, and conclusions or recommendations expressed in this material are those of the author(s) and do not necessarily reflect the views of the funders.

\section{REFERENCES}

Allahbakhsh, M., Benatallah, B., Ignjatovic, A., Motahari-Nezhad, H. R., Bertino, E., and Dustdar, S. (2013). Quality control in crowdsourcing systems: Issues and directions. IEEE Internet Computing, 17(2):76-81. 00152.

Alpaydin, E. (2020). Introduction to Machine Learning. The MIT Press, 4th edition.

Berinsky, A. J., Huber, G. A., and Lenz, G. S. (2012). Evaluating online labor markets for experimental research: Amazon.com's mechanical turk. Political Analysis, 20(3):351-368.

Bernstein, M. S., Little, G., Miller, R. C., Hartmann, B., Ackerman, M. S., Karger, D. R., Crowell, D., and Panovich, K. (2015). Soylent: a word processor with a crowd inside. Communications of the ACM, 58(8):85-94. 00607.

Bevelander, K. E., Kaipainen, K., Swain, R., Dohle, S., Bongard, J. C., Hines, P. D. H., and Wansink, B. (2014). Crowdsourcing Novel Childhood Predictors of Adult Obesity. PLOS ONE, 9(2):e87756. 00019.

Bongard, J. C., Hines, P. D., Conger, D., Hurd, P., and Lu, Z. (2013). Crowdsourcing predictors of behavioral outcomes. IEEE Transactions on Systems, Man, and Cybernetics: Systems, 43(1):176-185. 00024.

Breiman, L. (2001). Random Forests. Machine Learning, 45(1):5-32.

Chandler, J., Rosenzweig, C., Moss, A. J., Robinson, J., and Litman, L. (2019). Online panels in social science research: Expanding sampling methods beyond mechanical turk. Behavior research methods, 51(5):2022-2038.

Cheng, J. and Bernstein, M. S. (2015). Flock: Hybrid crowd-machine learning classifiers. In Proceedings of the 18th ACM Conference on Computer Supported Cooperative Work \&\#38; Social Computing, CSCW'15, pages 600-611, New York, NY, USA. ACM.

Chilton, L. B., Little, G., Edge, D., Weld, D. S., and Landay, J. A. (2013). Cascade: Crowdsourcing taxonomy creation. In Proceedings of the SIGCHI Conference on Human Factors in Computing Systems, pages 1999-2008. ACM. 00117.

Crandall, J. W., Oudah, M., Tennom, Ishowo-Oloko, F., Abdallah, S., Bonnefon, J.-F., Cebrian, M., Shariff, A., Goodrich, M. A., and Rahwan, I. (2018). Cooperating with machines. Nature Communications, 9(1):233. 
David, H. (2015). Why are there still so many jobs? the history and future of workplace automation. Journal of Economic Perspectives, 29(3):3-30.

Domingos, P. (2012). A few useful things to know about machine learning. Communications of the ACM, 55(10):78-87.

Dontcheva, M., Gerber, E., and Lewis, S. (2011). Crowdsourcing and creativity. In CHI 2011: Crowdsourcing Workshop. 00010.

Ellsberg, D. (1961). Risk, ambiguity, and the Savage axioms. The Quarterly Journal of Economics, pages 643-669.

Fails, J. A. and Olsen Jr, D. R. (2003). Interactive machine learning. In Proceedings of the 8th international conference on Intelligent user interfaces, pages 39-45.

Feurer, M., Klein, A., Eggensperger, K., Springenberg, J., Blum, M., and Hutter, F. (2015). Efficient and robust automated machine learning. In Advances in Neural Information Processing Systems, pages 2962-2970.

Frank, M. R., Autor, D., Bessen, J. E., Brynjolfsson, E., Cebrian, M., Deming, D. J., Feldman, M., Groh, M., Lobo, J., Moro, E., Wang, D., Youn, H., and Rahwan, I. (2019). Toward understanding the impact of artificial intelligence on labor. Proceedings of the National Academy of Sciences, 116(14):6531-6539.

Hsueh, P.-Y., Melville, P., and Sindhwani, V. (2009). Data quality from crowdsourcing: a study of annotation selection criteria. In Proceedings of the NAACL HLT 2009 workshop on active learning for natural language processing, pages 27-35. Association for Computational Linguistics.

Hutter, F., Kotthoff, L., and Vanschoren, J. (2019). Automated Machine Learning. Springer.

Kamar, E., Hacker, S., and Horvitz, E. (2012). Combining human and machine intelligence in large-scale crowdsourcing. In Proceedings of the 11th International Conference on Autonomous Agents and Multiagent Systems-Volume 1, pages 467-474.

Kittur, A. (2010). Crowdsourcing, collaboration and creativity. XRDS: crossroads, the ACM magazine for students, 17(2):22-26.

Lease, M. (2011). On quality control and machine learning in crowdsourcing. In Proceedings of the 11th AAAI Conference on Human Computation, AAAIWS'11-11, pages 97-102. AAAI Press.

Li, Q., Ma, F., Gao, J., Su, L., and Quinn, C. J. (2016). Crowdsourcing high quality labels with a tight budget. In Proceedings of the Ninth ACM International Conference on Web Search and Data Mining, pages 237-246. ACM. 00004.

McAndrew, T. C., Guseva, E., and Bagrow, J. P. (2017). Reply \& Supply: Efficient crowdsourcing when workers do more than answer questions. PLOS ONE, 12(8):e69829.

Negahban, S., Oh, S., and Shah, D. (2017). Rank centrality: Ranking from pairwise comparisons. Operations Research, 65(1):266-287.

Salganik, M. J. and Levy, K. E. (2015). Wiki surveys: Open and quantifiable social data collection. PLOS ONE, 10(5): 0123483.

Schenk, E. and Guittard, C. (2009). Crowdsourcing: What can be outsourced to the crowd, and why. In Workshop on Open Source Innovation, Strasbourg, France, volume 72.

Scholer, F., Turpin, A., and Sanderson, M. (2011). Quantifying test collection quality based on the consistency of relevance judgements. In Proceedings of the 34th international ACM SIGIR conference on Research and development in Information Retrieval, pages 1063-1072. ACM. 00053.

Siangliulue, P., Arnold, K. C., Gajos, K. Z., and Dow, S. P. (2015). Toward collaborative ideation at scale: Leveraging ideas from others to generate more creative and diverse ideas. In Proceedings of the 18th ACM Conference on Computer Supported Cooperative Work \& Social Computing, pages 937-945. ACM.

Swain, R., Berger, A., Bongard, J., and Hines, P. (2015). Participation and contribution in crowdsourced surveys. PLOS ONE, 10(4):e0120521. 00004.

Teevan, J., Iqbal, S. T., and von Veh, C. (2016). Supporting Collaborative Writing with Microtasks. In Proceedings of the 2016 CHI Conference on Human Factors in Computing Systems, CHI '16, pages 2657-2668, New York, NY, USA. ACM.

Tversky, A. and Kahneman, D. (1974). Judgment under uncertainty: Heuristics and biases. Science, 185(4157):1124-1131.

Valiant, L. G. (1984). A theory of the learnable. Communications of the ACM, 27(11):1134-1142.

Vanschoren, J., van Rijn, J. N., Bischl, B., and Torgo, L. (2014). Openml: Networked science in machine learning. SIGKDD Explor. Newsl., 15(2):49-60. 
${ }_{636}$ Vapnik, V. (2013). The nature of statistical learning theory. Springer science \& business media.

637 Wagy, M. D., Bongard, J. C., Bagrow, J. P., and Hines, P. D. H. (2017). Crowdsourcing predictors of 638 residential electric energy usage. IEEE Systems Journal, PP(99):1-10.

639 Yang, Q., Suh, J., Chen, N.-C., and Ramos, G. (2018). Grounding interactive machine learning tool design

640 in how non-experts actually build models. In Proceedings of the 2018 Designing Interactive Systems

${ }_{641}$ Conference, DIS '18, pages 573-584, New York, NY, USA. Association for Computing Machinery. 